\title{
An Iterative Technique for Real-Time Tracking of Power System Harmonics
}

\author{
T. S. Sidhu*, M. R. D. Zadeh*, P. J. Pooranalingam** and Yong-Taek $\mathrm{OH}^{\dagger}$
}

\begin{abstract}
An iterative technique based on orthogonal filters and frequency tracking is proposed to estimate harmonic components in power systems. The technique uses frequency interpolation to estimate fundamental frequency and harmonics when the nominal frequency of the signal is a non-integer value. Due to the number of computations involved during the generation of filter coefficients, an offline computation is suggested. Beneficial features of the proposed technique include fixed sampling rate and fixed data window size. The performance of the proposed technique is examined by simulating different power system operating conditions and evaluating the data from these simulations. A technique based on Fast Fourier Transform is also used to estimate the harmonic components for all the simulated signals. These estimates are compared with those obtained from the proposed technique. Results show that the proposed technique can converge to the accurate fundamental frequency and therefore, provide accurate harmonic components even when the fundamental frequency is not equal to the nominal frequency.
\end{abstract}

Keywords: Iterative technique, Frequency tracking, Harmonic estimation, FFT

\section{Introduction}

The widespread applications of power electronic devices have increased the harmonic distortion in power system voltage and current waveforms. Advances in semiconductor device technology have fueled a revolution in power electronics over the past decade, and there is every indication that this trend will continue. There is, however, concern on distortion from the non-fundamental periodic voltages and currents resulting from the steady state operation of nonlinear elements connected to the system [1], and during disturbances and faults.

Steady state harmonic distortions are caused by nonlinear devices in a power system. In high voltage generating, transmission, and distribution systems, there are many possibilities to generate transient harmonics during faults and disturbances. Nonlinear branches, such as magnetizing reactance in transformers, can produce harmonics as a consequence of saturation effects during faults and disturbances. The severity of transient corruption depends on network type, fault type, fault location, fault sequence, switching type, sequence impedance ratio $\left(Z_{0} / Z_{1}\right)$, fault incident angle, and so on. [2].

Due to the increased presence of harmonics and its effects

$\dagger \quad$ Corresponding author: Dept. of Electrical and Electronic Engineering, Korea University of Technology and Education, Korea. (Ytoh@kut.ac.kr)

* Dept. of Electrical and Computer Engineering, University of Western Ontario,Canada,(tsidhu@uwo.ca, m.zadeh@gmail.com)

* Dept. of Electrical and Electronic Engineering, University of Saskatchewan, Canada. (pjp530@engr.usast.ca)

Received: November 16, 2009; Accepted: Febryary 8, 2011 in the modern power system, the necessity of real-time monitoring and analysis of variations of harmonics is widely acknowledged. A survey of related literature revealed that most studies conducted on harmonic estimations have limitations, particularly during frequency drift and presence transients. Furthermore, some of the recently proposed algorithms demand large amounts of computations.

The objective of this paper is to develop a technique for real-time estimation of power system harmonics and to simulate and test this technique using MATLAB. The technique is designed to provide accurate estimations for distorted waveforms and frequency drifts normally encountered in power systems. The implementation of this technique in digital signal processor (DSP)-based platform is in progress at the University of Western Ontario.

Section II presents an overview of the previously proposed algorithms for harmonic estimations. A new iterative technique for harmonic estimation is developed and described in Section III. In Section IV, the technique is simulated and tested using the voltage and current waveforms obtained from simulations in which various power system conditions were considered. Results obtained using the simulated data for various case studies are illustrated and discussed in this section. Estimates obtained from the proposed technique are compared with those obtained a technique based on Fast Fourier Transform (FFT) and error analyses are conducted.

\section{Harmonic Estimation Techniques}

A number of algorithms for estimating harmonics have 
been proposed in the past. These algorithms use a variety of approaches, including Discrete Fourier Transform (DFT) and FFT [3], Kalman filtering [4], and Artificial Neural Networks [5]. This section briefly reviews these techniques. A short analysis of the pitfalls of these techniques and their effect on harmonic estimates is also presented.

\subsection{Discrete and Fast Fourier Transform}

Most harmonic analysis algorithms are based on FFT and DFT to obtain the voltage and current frequency spectrum from discrete time samples.

FFT is a very efficient algorithm for evaluating Fourier transform. This algorithm becomes considerably more efficient when a large number of data in a discrete sequence need to be transformed into frequency domain. For example, the total number of calculations for $\mathrm{N}$ point DFT is $\mathrm{N}^{2}$, and for FFT, it is $\mathrm{Nlog}_{2} \mathrm{~N}$. In FFT, the number of sampled values in a cycle should be chosen to be a power of 2 .

There are two major pitfalls in the application of FFT: picket-fence effect and spectral leakage [6]. The picketfence effect occurs when the analyzed waveform includes a frequency that is not an integer times the fundamental frequency. During faults or disturbances in the power systems, transients produce decaying DC components which can lead to estimation errors. Spectral leakage arises due to the truncation of the sequence, such that a fraction of a cycle exists in the analyzed waveform. Modern data acquisition systems usually employ fixed sampling rate and data window. If the sampled waveform does not contain an integer number of samples per integer number of cycles, the results of the DFT and FFT will include errors.

The resulting errors are known as spectral leakage [6]. The two main errors caused by spectral leakage are as follows:

(1) A reduction in main lobe output.

(2) Contributions from neighboring harmonics.

A modern data acquisition system usually has a fixed sampling rate; thus, the window length is constant, which leads to spectral leakage. The magnitude of spectral leakage error in harmonic estimations depends on the following:

(1) The deviation from the actual to the nominal fundamental frequency;

(2) The shape of the filter characteristic;

(3) The size of the neighboring harmonics; and

(4) The desired harmonic number.

For example, a $0.4 \mathrm{~Hz}$ deviation in the fundamental frequency will result in a $4 \mathrm{~Hz}$ deviation at the $10^{\text {th }}$ harmonic. Thus, the spectral leakage error will worsen as the harmonic number increases.

\subsection{Kalman Filter}

The Kalman filter [7] is a recursive filter whose output depends on both present and past inputs. It differs from other filtering algorithms used in power systems such that its gain coefficients vary with time.

The design of the Kalman filter is based on the statistical property of the signal to be processed. The time-varying filter coefficients, called Kalman gains, are calculated to minimize the square of the expected errors between the values of the actual estimated systems states.

The Kalman filter performs optimally if the statistical properties of the currents and voltages being processed are known and are used in designing the filter. The filter produces sub-optimal phasor estimates because the statistical properties are not known and are only estimated.

The Kalman filter theory is effective only if the change in the voltage phasor occurs at successive instants, the noise components have zero means and white, and their amplitudes have Gaussian distributions [7]. For inputs which satisfy these conditions, Kalman filter will give an optimal filter design. In practical situations, the variables do not satisfy these ideal conditions. For example, in real systems, the noise components are band limited and are, therefore, perfectly non-white. In addition, the signals are often correlated signals and are, therefore, not white. The random variables may not have strictly Gaussian distribution, but are assumed to be Gaussian when designing the Kalman filter. This factor can also cause error to Kalman filter-based estimations. The Kaman filter provides an output that is unacceptable for measurement because it cannot account for the non- $60 \mathrm{~Hz}$ components in the input signals. Selecting a larger value for the noise variance to account for all the non- $60 \mathrm{~Hz}$ components will slow the output [7].

The accuracy of Kalman filter outputs will be adversely affected if the components in the input signal have not been modeled. Fundamental frequency variations in power systems are common and their deviation is unpredictable. Since the Kalman filter is not modeled for frequency variations, during the fundamental frequency variations, fundamental frequency and harmonic component estimations will have errors similar to the DFT technique.

\subsection{Artificial Neural Network}

Lai, Tse, Chan, and So proposed a neural network based technique [5]. They used Hopfield-type feedback neural networks for real-time harmonic estimation.

The proposed neural network implementation simultaneously determines the supply frequency variation, the fundamental amplitude/phase variation, as well as the harmonics amplitude/phase variation. Its distinctive feature is that the supply frequency variation is handled separately from the amplitude/phase variations.

The main problem of these techniques is the number of computations involved during the estimation of fundamental frequency and harmonic phase values. Effective online implementation of these techniques may be very expensive or even impossible to implement using the currently available DSP processor due to the number of computations involved. 


\section{The Proposed technique}

The theoretical basis of the proposed technique is derived from an error analysis of the previous techniques which assume that the frequency of a power system is fixed. The proposed technique uses an iterative method to track the fundamental frequency of a power system and the estimated frequency to select the appropriate filters for computing various harmonics. On-line and off-line computations involved in implementing the proposed technique are also described. Practical issues in the implementation of the proposed technique using a microprocessor-based system are discussed.

\subsection{Error Analysis of Different Techniques}

From the previous discussion, accurate estimates will clearly be obtained if appropriate orthogonal filters are used as the frequency of power system changes. The filter designed by assuming the prevailing fundamental frequency should be used for harmonic estimation, which means that the prevailing fundamental frequency must also be estimated.

Furthermore, the window length should not change for ease of implementation. The sampling rate should be kept constant to allow easy implementation and use of synchronized sampling if needed.

\subsection{The Proposed Technique}

The technique consists of two parts. The first part tracks the power system's fundamental frequency, and the second part ascertains the power systems harmonics based on the prevailing fundamental frequency. The method used for tracking the power system frequency is based on an iterative procedure proposed by the author [8]. The technique considers that samples of a signal having a fundamental frequency of $f_{0} \mathrm{~Hz}$ are taken at a pre-specified sampling rate. Designing orthogonal filters to extract the real and imaginary parts of the fundamental frequency component of the signal is possible.

The real and imaginary parts, $V_{r n}$ and $V_{i n}$, computed using samples corresponding to the $n^{\text {th }}$ data window can be used to estimate the phase angle, $\theta_{n}$, of the fundamental frequency phasor corresponding to the $n^{\text {th }}$ window by using (1).

$$
\theta_{n}=\tan ^{-1}\left(\frac{V_{i n}}{V_{r n}}\right)
$$

where $V_{r n}$ is the real part of the fundamental phasor, and $V_{i n}$ is the imaginary part of the fundamental phasor.

As the next sample arrives, the data window is advanced by one sample. The phase angle, $\theta_{n+1}$ of the fundamental frequency phasor corresponding to the $(n+1)^{\text {th }}$ data window can be computed using the data from the $(n+1)^{\text {th }}$ window and the coefficients of the orthogonal filters. The phase angle difference, $\left(\theta_{n+I^{-}} \theta_{n}\right)$, represents the rotation of the phasor in one sampling interval. The rotation for a phasor having a fundamental frequency of $f_{0}$ will be equal to

$$
\theta_{n+1}-\theta_{n}=\frac{2 \pi f_{0}}{f_{s}}
$$

where $f_{s}$ is the sampling rate.

Eq. (2) is only true if phase angles $\theta_{n}$ and $\theta_{n+1}$ are computed using the orthogonal filters designed by assuming the fundamental frequency of the signal as $f_{0}$. In spite of this, (2) forms the basis for the frequency estimation and estimates of the frequency, $f^{\wedge}$, which can be obtained as follows:

$$
f^{\wedge}=\frac{2 \pi}{\left(\theta_{n+1}-\theta_{n}\right) \times f_{s}}
$$

where $f^{\wedge}$ is the estimated frequency.

One of the following two situations can exist:

(a) The estimated frequency is equal to the fundamental frequency assumed for designing the orthogonal filters used to compute the phase angles $\theta_{n}$ and $\theta_{n+l}$. This means that the estimated frequency is also the fundamental frequency of the signal.

(b) The estimated frequency is not equal to the fundamental frequency assumed for designing the orthogonal filters. This means that the estimated frequency is not the fundamental frequency of the signal. To achieve the previous situation, the phase angles need to be recalculated using the orthogonal filters designed by assuming that the fundamental frequency is equal to the fundamental frequency of the signal. This can be achieved using an iterative procedure as follows:

(1) Design new orthogonal filters by assuming that the fundamental frequency of the signal is equal to the latest estimate of the frequency obtained from (3).

(2) Compute the phase angles $\theta_{n}$ and $\theta_{n+1}$ using the orthogonal filters designed in Step 1 and the samples corresponding to the data windows $n$ and $(n+1)$.

(3) Estimate the frequency using (3) and the phase angles computed in Step 2.

(4) Check if the estimated frequency from Step 3 is equal to the fundamental frequency assumed for designing the filters in Step 1. If it is, the estimated frequency in Step 3 is the fundamental frequency of the signal; otherwise, the process reverts to Step 1.

An initial estimate of the frequency is assumed in starting the procedure for the first time. It is recommended in [8] that the initial estimate should be equal to the nominal frequency, i.e., $60 \mathrm{~Hz}$. Once the prevailing fundamental frequency is estimated, orthogonal filters are then designed to extract the desired harmonic components of the esti- 
mated fundamental frequency.

\subsection{Practical Issues}

As discussed previously, the estimation process may require the design of new orthogonal filters after every iteration. The design of filters requires a considerable amount of computations. These computations may not be completed within one sampling interval, which is available for performing calculations. Therefore, the filters should be designed off-line and their coefficients can be stored for use in estimating the frequency and harmonics.

The iterative procedure for estimating the frequency is terminated when the estimated frequency obtained from (3) is equal to the fundamental frequency assumed for designing orthogonal filters. In practice, a margin should be allowed to account for errors arising from truncations during calculations, interpolations, and data acquisitions, among others.

The number of iterations that can be conducted in one sampling interval and the number of harmonics that can be estimated will be limited by the capability of a digital processor. As shown in [8], the number of iterations should be kept equal to five to achieve faster convergence during a step change in the frequency. However, if a digital processor cannot conduct five iterations in one sampling interval, the number of iterations per sampling interval can be set at less than five depending on the capability of the processor. This may result in one or two additional sampling intervals for convergence in situations where there is a step change in the frequency, or where the initial estimate is not very close to the signal frequency.

\subsection{Computations}

Off-line calculations include the design of orthogonal filters for extracting the fundamental frequency component and desired harmonic components. These filters are designed by assuming a fundamental frequency of the signal equal to $40-70 \mathrm{~Hz}$ in the steps of $1 \mathrm{~Hz}$.

The coefficients of the resulting sine and cosine filters are stored in the form of a look-up table that is used during on-line calculations.

This means that the coefficients of filters corresponding to the abovementioned frequencies are directly available. For filter coefficients corresponding to other frequencies, linear interpolation must be conducted. This arrangement facilitates the estimation of frequencies that exist in a power system and is accurate. It can be extended by storing the coefficients of filters covering a wider range.

\section{Simulation Studies}

Various types of signals were synthesized and discretized using MATLAB. These data were used to verify the validity and accuracy of the technique. Harmonic estimates obtained from the proposed technique are shown in this section. The results were also compared with those obtained from the commonly used FFT-based technique.

\subsection{Simulation Modules}

A program, the data generation module, was developed for synthesizing and discretizing the test signals at a specified sampling rate. This program accepts the user-supplied parameters of the signal to be synthesized, including the fundamental frequency of the signal and the magnitudes and phase angles of various harmonic components. The program stores the discretized data in a file for use by other programs.

In the first program, the FFT module was developed to estimate the harmonic components of a signal. This module uses the well-known FFT technique. In another program, the proposed technique module implemented the proposed technique. This module conducts off-line and on-line computations and estimates the fundamental frequency and harmonic components of the signal. The following parameters were chosen for the current study:

- Sampling rate of $3840 \mathrm{~Hz}$

- Data window for the proposed technique: 129 samples

- Data window for the FFT technique: 128 samples

- Fundamental frequency range: 50 to $70 \mathrm{~Hz}$

- Number of harmonics: 25

The time reference coincided with the middle of the window for the design of Least Error Squares orthogonal filters [8].

\subsection{Tests and Results}

A variety of signals that may be encountered in a power system were synthesized and discretized using the data generation module. The discretized data were given to the FFT module and the proposed technique module to estimate the harmonic components of the signals. Five cases have been examined in this paper. Each case was discussed and the transient responses of the proposed technique and FFT were presented in graphs. The maximum steady state errors which resulted in odd harmonics are illustrated for all the five cases in Table 1 . The harmonics components with zero magnitude and absolute error are shown in the table while the error in percentage is given for the harmonics with non-zero magnitude.

\section{(1) Distorted Signal with a Nominal Fundamental Fre- quency (Case 1)}

Eq. (4) is a distorted signal with a nominal fundamental frequency of $60 \mathrm{~Hz}$.

$$
\begin{aligned}
& i(t)=100 \cos (\omega t+\pi / 6)+4 \cos (3 \omega t+\pi / 4)+ \\
& 12 \cos (5 \omega t+13 \pi / 36)+4 \cos (7 \omega t+\pi / 4)+ \\
& 3 \cos (11 \omega t+\pi / 6)+15 \cos (13 \omega t+\pi / 9)+3 \cos (19 \omega t)
\end{aligned}
$$


where $\omega=2 \pi 60$ radians/second.

The harmonic estimates by both techniques are accurate because the drift between the nominal frequency and the fundamental frequency is zero; thus, FFT estimates are not affected by spectrum leakage. The proposed technique also provides accurate estimates because it first converges to the fundamental frequency and then estimates the harmonics based on the estimated fundamental frequency.

\section{(2) Distorted Signal with an Off-Nominal Fundamental Frequency (Case 2)}

Eq. (5) is a distorted signal with an off-nominal fundamental frequency of $59 \mathrm{~Hz}$.

$$
\begin{aligned}
& i(t)=100 \cos (\omega t+\pi / 6)+10 \cos (3 \omega t+\pi / 7)+ \\
& 8 \cos (5 \omega t+\pi / 6)++8 \cos (7 \omega t+3 \pi / 2)+ \\
& 6 \cos (11 \omega t+5 \pi / 4)+5 \cos (13 \omega t+2 \pi / 3)+ \\
& 3 \cos (19 \omega t)
\end{aligned}
$$

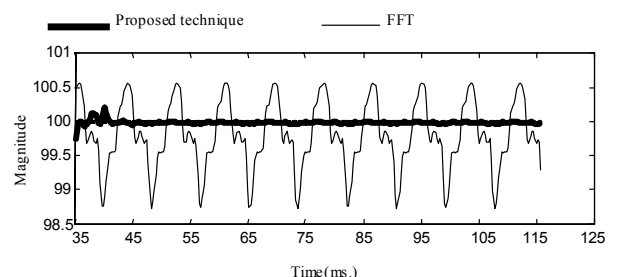

(a) Fundamental frequency, actual value $=100$

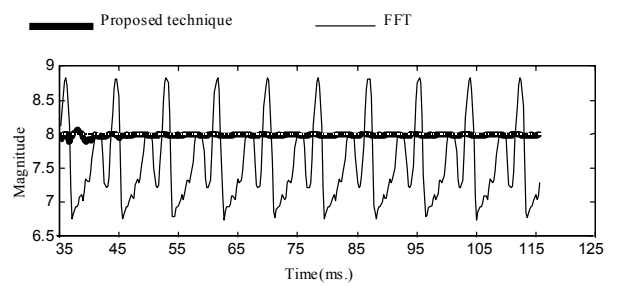

(b) $5^{\text {th }}$ harmonic, actual value $=8$

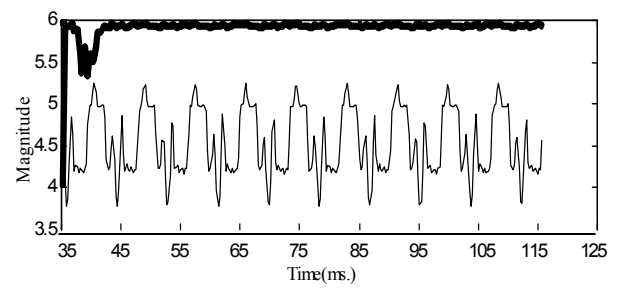

(c) $11^{\text {th }}$ harmonic, actual value $=6$

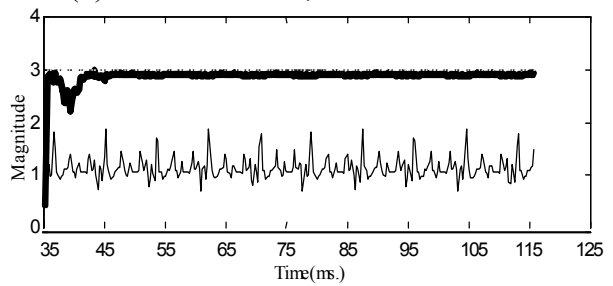

(d) $19^{\text {th }}$ harmonic, actual value $=3$

Fig. 1. Magnitude estimates of the harmonic components of a highly distorted current signal (Eq. (4.2)) with a fundamental frequency of $58.9 \mathrm{~Hz}$.

\section{(3) Distorted Signal with Dynamic Frequency Oscillation (Case 3)}

To demonstrate fast detection and convergence of the proposed technique, a current signal with a rising or decaying fundamental frequency was selected in which the fundamental frequency of the current signal oscillates by \pm 3 $\mathrm{Hz}$ and frequency of oscillations is $1 \mathrm{~Hz}$. The current signal was synthesized and discretized for the abovementioned condition, and is described by Eq. (6).

$$
\begin{aligned}
& i(t)=100 \sin v(2 \pi f(t))+5 \sin (6 \pi f(t)+\pi / 3)+ \\
& 10 \sin (10 \pi f(t)+\pi / 6)+7 \sin (14 \pi f(t))+ \\
& 5 \sin (22 \pi f(t)+\pi / 4)
\end{aligned}
$$

where $f(t)=\int(60+3 \sin (2 \pi t)) d t$.

The discretized data were given to the FFT module and the proposed technique module to estimate the harmonics. Fig. 4.4 shows the results obtained from both modules.

In Fig. 2(a), the proposed technique can accurately and efficiently trace the variations in the fundamental frequency with a delay of $20 \mathrm{~ms}$. Thus, the proposed technique estimates harmonics accurately based on the estimated fundamental frequency. The tests were repeated using other decay and rise rates, and similar results were obtained.

Fig. 2(b)-(g) and Table 1 show the harmonic estimates using the FFT and the proposed technique. Due to the continuous drift in frequency, the FFT-based estimation error change and the accurate values are estimated only when the input fundamental frequency is equal to the assumed frequency of $60 \mathrm{~Hz}$. On the other hand, the proposed technique provides accurate estimates at all times because the proposed technique tracks the signal frequency and is not affected by frequency drift.

\subsection{Distorted Signal with Decaying Magnitude of the Fundamental Frequency Component (Case 4)}

Eq. (7) shows the distorted current signal which has a fundamental frequency of $59 \mathrm{~Hz}$ and the peak value of the fundamental frequency component decaying exponentially from $100 \mathrm{~V}$.

$$
\begin{aligned}
& i(t)=100 e^{-\lambda t} \cos (\omega t+\pi)+10 \cos (3 \omega+\pi / 5)+ \\
& 8 \cos (5 \omega t+4 \pi / 3)+8 \cos (7 \omega t+\pi / 3)+ \\
& 6 \cos (11 \omega t+3 \pi)+5 \cos (13 \omega t+2 \pi / 3)+3 \cos (19 \omega t)
\end{aligned}
$$

where $\lambda=2$, and $\omega=2 \pi 59$ radians/second.

In this case, the proposed technique follows the actual value with a delay of $23 \mathrm{~ms}$ [see Fig. 3(a)]. Although the FFT-based technique follows the decaying fundamental frequency phasor fairly closely, as shown in Fig. 3(b), the higher order harmonic estimates are in error due to the fundamental frequency deviation from the nominal frequency. 


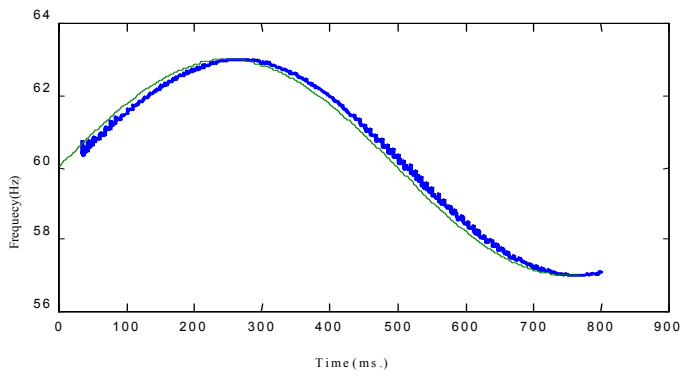

(a) Fundamental frequency estimate

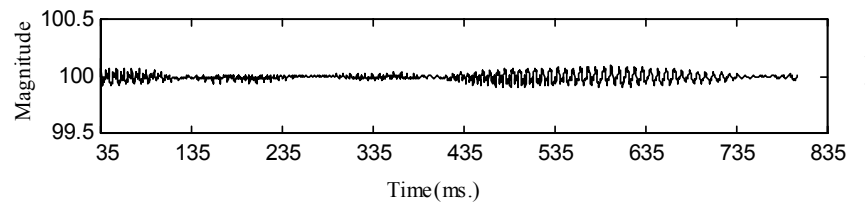

(b) Fundamental frequency (proposed technique

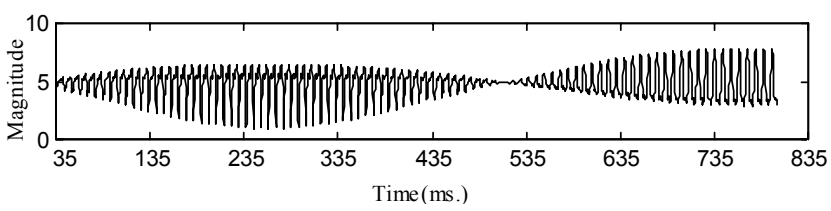

(d) $3^{\text {rd }}$ harmonic (proposed technique)

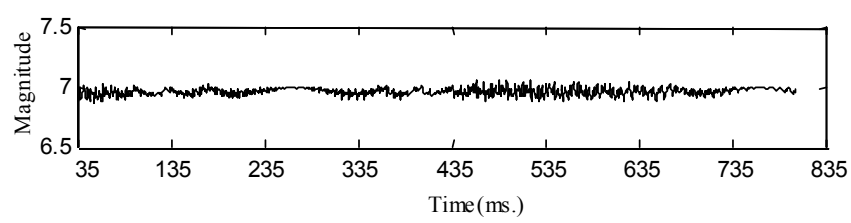

(f) $7^{\text {th }}$ harmonic (proposed technique)

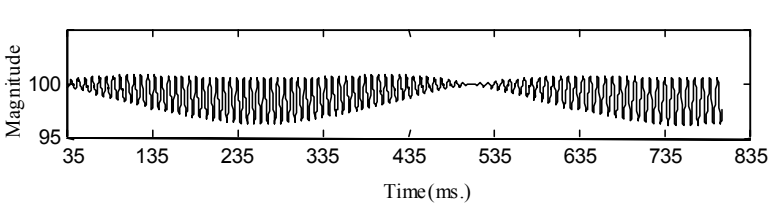

(c) Fundamental frequency (FFT technique)

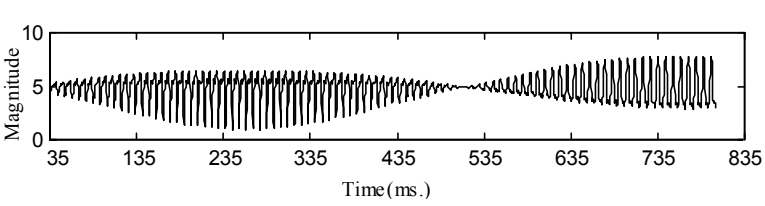

(e) $3^{\text {rd }}$ harmonic (FFT technique)

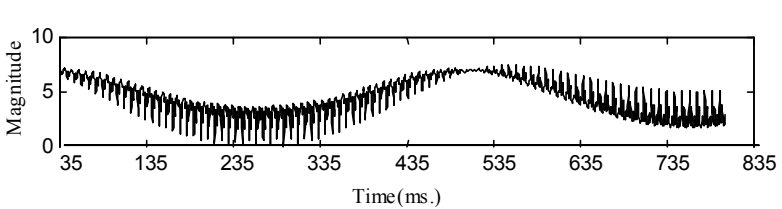

(g) $7^{\text {th }}$ harmonic (FFT technique)

Fig. 2. Magnitude estimates of the harmonic components of a distorted current signal in which fundamental frequency of $1 \mathrm{~Hz}$.
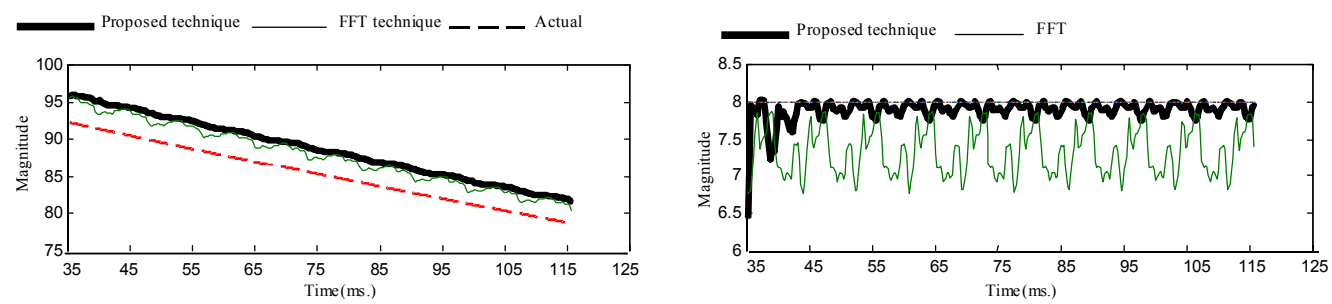

Fig. 3. Magnitude estimates of the harmonic components of a highly distorted current signal (Eq. (4.5)) with a decaying fundamental frequency component.

By repeating the testing for various frequency drifts and decaying rates, the proposed technique provides acceptable results, while the error increases with the frequency drift and with the harmonic component in the FFT-based technique.

\subsection{Abrupt Change in Harmonic Magnitude (Case 5)}

A voltage signal comprising of typical tripplen harmonic was synthesized and discretized in which a third harmonic changes abruptly. A voltage signal described by (8) was considered for simulation. The peak value of the $3^{\text {rd }}$ harmonic component in this signal changed abruptly from 10 to $8 \mathrm{~V}$.

$$
\begin{aligned}
& v(t)=100 \cos (\omega t+\pi)+5 \cos (2 \omega t+\pi / 4)+ \\
& A_{3} \cos (3 \omega t+\pi / 5)+8 \cos (5 \omega t+\pi / 3)+ \\
& 4 \cos (6 \omega t+\pi / 2)+8 \cos (7 \omega t+2 \pi / 3) \\
& +3 \cos (19 \omega t+2 \pi / 5)
\end{aligned}
$$



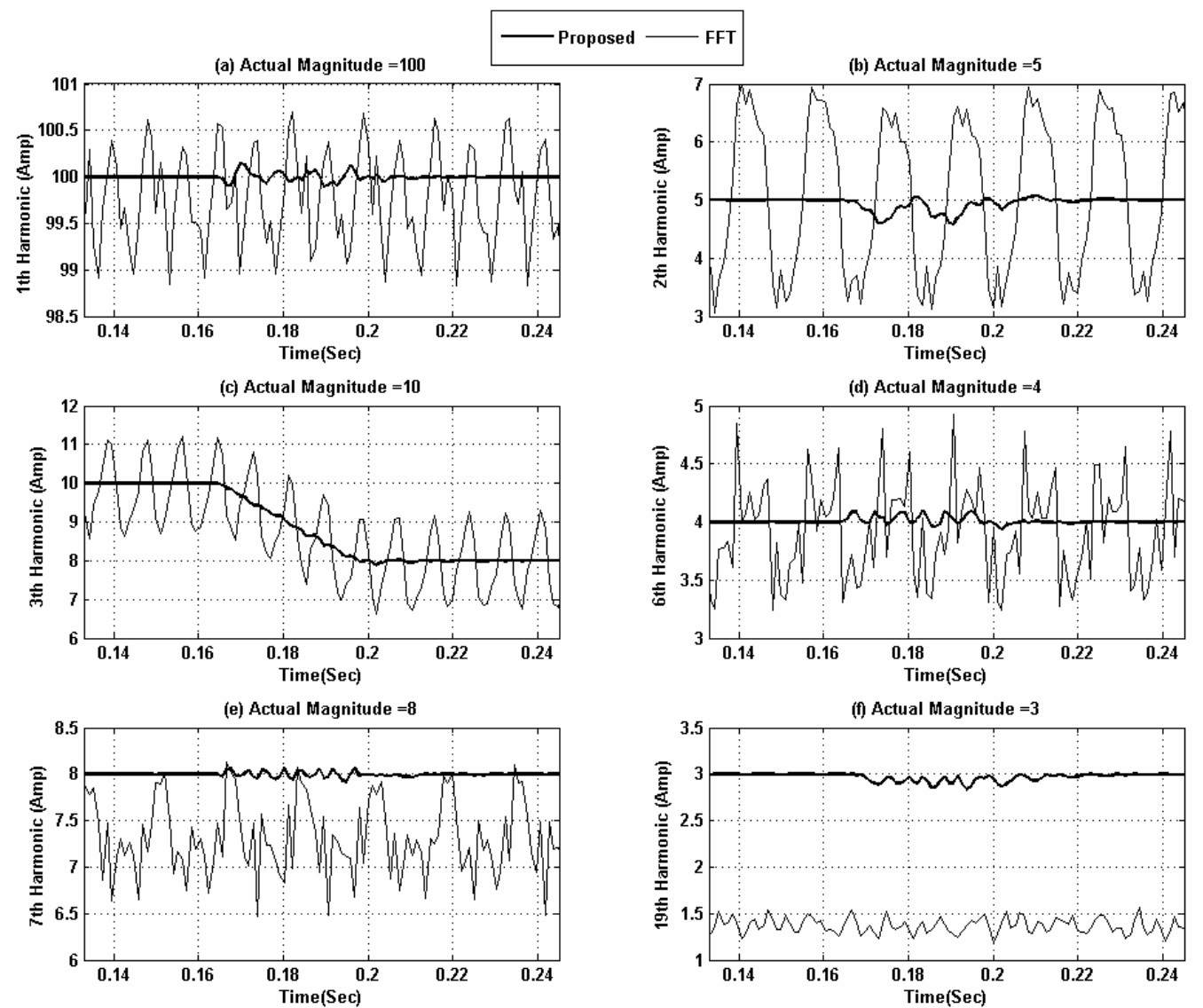

Fig. 4. Magnitude estimates of the harmonic components of a signal where the $3^{\text {rd }}$ harmonic component changes abruptly.

Table 1. Maximum Steady State Error for studied cases

\begin{tabular}{|c|c|c|c|c|c|c|c|c|c|c|c|}
\hline & & $1^{\mathrm{st}}$ & $3^{\text {rd }}$ & $5^{\text {th }}$ & $7^{\text {th }}$ & $9^{\text {th }}$ & $11^{\text {th }}$ & $13^{\text {th }}$ & $15^{\text {th }}$ & $17^{\text {th }}$ & $19^{\text {th }}$ \\
\hline \multirow{2}{*}{ Case 1} & Proposed Technique & 0 & 0 & 0 & 0 & 0 & 0 & 0 & 0 & 0 & 0 \\
\hline & FFT & 0 & 0 & 0 & 0 & 0 & 0 & 0 & 0 & 0 & 0 \\
\hline \multirow{2}{*}{ Case 2} & Proposed Technique & 0.1 & 0.3 & 0.9 & 1.1 & 0.1 & 2.7 & 3.4 & 0.1 & 0.1 & 6.7 \\
\hline & FFT & 1.5 & 8.1 & 18.1 & 16.6 & 1.3 & 38.0 & 42.7 & 0.8 & 0.6 & 70.4 \\
\hline \multirow{2}{*}{ Case 3} & Proposed Technique & 0.3 & 5.7 & 2.7 & 4.3 & 0.2 & 9.2 & 0.3 & 0.2 & 0.2 & 0.2 \\
\hline & FFT & 4.2 & 76.3 & 55.2 & 93.4 & 2.1 & 99.8 & 1.2 & 0.9 & 0.7 & 0.6 \\
\hline \multirow{2}{*}{ Case 4} & Proposed Technique & 0.9 & 1.9 & 1.4 & 3.8 & 0.2 & 5.7 & 0.2 & 0.1 & 0.1 & 16.2 \\
\hline & FFT & 1.4 & 7.1 & 14.0 & 15.7 & 1.3 & 27.8 & 0.9 & 0.6 & 0.6 & 64.0 \\
\hline \multirow{2}{*}{ Case 5} & Proposed Technique & 0.1 & 1.3 & 2.5 & 0.6 & 0.1 & 0.0 & 0.0 & 0.0 & 0.0 & 4.2 \\
\hline & FFT & 1.3 & 16.4 & 30.1 & 25.1 & 1.0 & 0.6 & 0.5 & 0.4 & 0.5 & 61.1 \\
\hline
\end{tabular}

where $\omega=2 \pi 59$ radians/second. $\mathrm{A}_{3}$ is equal to 10 for $0<\mathrm{t}$ $<165 \mathrm{~ms}$, and equal to 8 for $\mathrm{t}>165 \mathrm{~ms}$.

Fig. 4(a)-(f) and Table 1 show the response of the proposed technique and the FFT technique with an abrupt change in the $3^{\text {rd }}$ harmonic. These figures illustrate that the impact on other harmonic estimates by the proposed technique due to abrupt change in the $3^{\text {rd }}$ harmonic is negligible. The proposed technique tracks the new $3^{\text {rd }}$ harmonic value within $33.59 \mathrm{msec}(129 / 3840 \mathrm{sec})$. The FFT-based technique tracks the changed value; however, due to the frequency drift, it has an error in the estimates of the higherorder harmonics.

\section{Conclusion}

The widespread applications of power electronic devices have increased the harmonic distortion in power system voltage and current waveforms. An examination of pitfalls in previously proposed techniques is presented. Examples are shown to indicate that blindly applying DFT and FFT may lead to incorrect harmonic estimations. The limitations of the Kalman filter and artificial neural networks-based techniques, and the difficulties faced during implementation are also discussed. A new iterative technique based on orthogonal filters and frequency tracking is developed to estimate harmonic components in power systems. This 
proposed technique uses frequency interpolation to estimate fundamental frequency and harmonics when the nominal frequency of the signal is a non-integer value. Due to the number of computations involved during the generation of the filter coefficients, off-line computations are proposed. The effect of various operating conditions on the performance of the proposed technique is also investigated. The technique provides accurate estimations without changing the data window length and sampling rate. The results obtained during the simulation and implementation studies indicate that the proposed technique has acceptable accuracy.

\section{Acknowledgements}

This work was supported by the Development of Prototype Multifunction IED for Internal Fault Protection of Large Generator of the Korea Institute of Energy Technology Evaluation and Planning (KETEP) grant funded by the Korea government Ministry of Knowledge Economy (2010T100100415).

\section{References}

[1] Thomas H. Ortmeyer, K.R.Chakravarthi, Aly A. Mahmoud, "The Effects of Power System Harmonics end Power System Equipment and Loads", IEEE Transactions on Power Apparatus and System, Vol. PAS-104, No. 9, pp. 2555-2560, September 1985.

[2] P.Sowa, Dutta Roy, M.Gopal “ Fault-Induced Harmonics Transients at the Relay Terminals", IEEE Region 10 International Conference on Connectivity in Energy, Vol. 3, 1998, pp. 440-443

[3] Timoty A. George and David Bones, "Harmonic Power Flow Determination Using the Fast Fourier Transform", IEEE Transactions on Power Delivery, Vol 6, No 3, April 1991.

[4] Adly A. Girgis, W. Bin Chang, Elham B.Markam, "A Digital Recursive Measurement Scheme for On-line Tracking of Power System Harmonics", Paper No. 91 WM 109-9 PWRD, 1991, IEEE/PES Winter Meeting, New York, New York, February 3, 1991.

[5] L.L. Lai, W.L. Chan, C.T. Tse, A.T.P. So, "Real-Time Frequency and Harmonic Evaluation using Artificial Neural Networks", IEEE Transactions on Power Delivery, Vol. 14, No. 1, pp. 52-57, January 1999.

[6] Adly A. Girgis, Fredric M. Ham, "A Quantitative Study of Pitfalls in the FFT", IEEE Transactions on Aerospace and Electronic Systems, Vol. AES-16, No. 4, pp. 434-439, July 1980.

[7] M. S., Sachdev, H. C. Wood, and N. G., Johnson, "Kalman Filtering Applied to Power System Measurements for Relaying”, IEEE Transactions on Power Apparatus and Systems, Vol. PAS-104, Dec.
1985, pp. 3565-3573.

[8] T.S. Sidhu, M.S. Sachdev, "An Iterative Technique for Fast and Accurate Measurement of Power System Frequency", IEEE Transactions on Power Delivery, Vol. 13, No. 1, pp.109-115, January 1998.

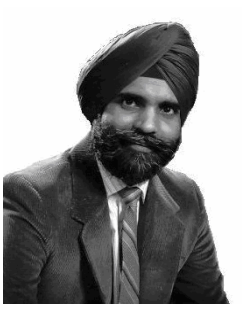

Tarlochan S. Sidhu received the B.E. (Hons) degree from the Punjabi University, Patiala, India, in 1979 and the M.Sc. and Ph.D. degrees from the University of Saskatchewan, Saskatoon, SK, Canada, in 1985 and 1989, respectively. He worked with the Regional Computer Center, Chandigarh, Punjab State Electricity Board, India, and Bell-Northern Research Ltd., Ottawa, Canada. From 1990 to 2002, he was with the Department of Electrical Engineering, University of Saskatchewan, Canada. Currently, he is a Professor and the Chair of the Electrical and Computer Engineering Department at the University of Western Ontario, London, Canada. He is also the NSERC/Hydro One Networks Senior Industrial Research Chair in Power Systems Engineering. Dr. Sidhu is a Fellow of the IEEE, the Institution of Engineers (India), the Institution of Engineering and Technology (U.K.) and Canadian Academy of Engineering. He is also a Registered Professional Engineer in the Province of Ontario and a Chartered Engineer in the U.K.

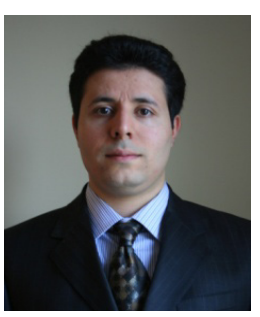

Mohammad R. Dadash Zadeh received B.S. and M.S. degrees in Electrical engineering from the University of Tehran in 2002 and 2005, respectively and received a $\mathrm{Ph}$. D. degree from the University of Western Ontario in 2009. He was a post doctoral fellow at the University of Western Ontario from May 2009 to January 2010. Currently, he is working with GE Digital Energy as an application engineer. His areas of interest include power system protection, control, and analysis.

P. J. Pooranalingam obtained his bachelor's degree from Russia and M.Sc. degree in electrical engineering from the University of Saskatchewan, Canada. Following completion of his degree, he worked for power-related industries including GE Multilin 


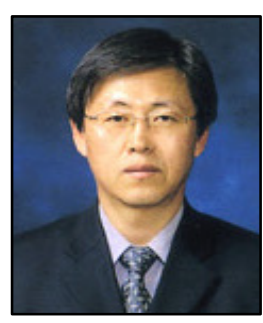

Yong-Taek Oh received his B.S degree from the Sungsil University, Korea in 1980 and the M.Sc and Ph.D degree Electrical Engineering from the Yonsei University, Korea in 1982 and 1987 respectively in Electrical Engineering. From 1987 to 1991, He was with the Computer Center of Korea Electric Power Corporation as a Section Chief. He joined the faculty of Korea University of Technology and education, Cheonan, Korea in 1991 where he is currently a professor in the school of Information Technology. His areas of research interest are power system protection and control, power system stability, fault analysis and computational intelligence and its application in power engineering. 